\title{
A Framework for Energy Efficient Collaborative Video Download and Sharing in Cloud Environment
}

\author{
G. Santhi \\ Assistant Professor \\ Department of Information Technology \\ Pondicherry Engineering College
}

\author{
P. Pavithraa, O. Nisha, G. Sujitha \\ Final Year, B.Tech \\ Department of Information Technology \\ Pondicherry Engineering College
}

\begin{abstract}
Video download using cloud services is one of the most popular applications in the field of mobile cloud computing. Reducing the energy consumption of the mobile devices is a major challenge in sustaining multimedia applications. In our proposed work, an adaptive framework is provided for group of users with common interest to collaboratively download a video file from the cloud and share it. In collaborative file download each node downloads only a segment of video file. These downloaded segments are reassembled and merged into a complete file and then it is shared to all users of the ad hoc group. Only the mobile nodes with relatively good battery level involve in video segment download whereas the nodes with low battery life remain futile. This greatly reduces the battery consumption of the mobile devices as a whole. Seamless connectivity is established between the mobile devices and the cloud infrastructure to smoothen the service.
\end{abstract}

\section{Keywords}

Collaborative file download; Ad hoc cloud; Seamless connectivity; File sharing; Energy saving.

\section{INTRODUCTION}

Cloud computing (CC) has become the hot trend since 2007 . Mobile Cloud Computing (MCC) is an emerging platform combining the mobile devices and the Cloud Computing to create a new infrastructure where the mobile devices can enjoy the benefits of Cloud Computing. By the datacenterknowledge.com [1] definition mobile cloud computing is defined as cloud computing extended by mobility and a new ad-hoc infrastructure based on mobile devices. Moreover the mobile users are provided with data storage and processing services on a cloud computing platform rather than on the mobile devices themselves.

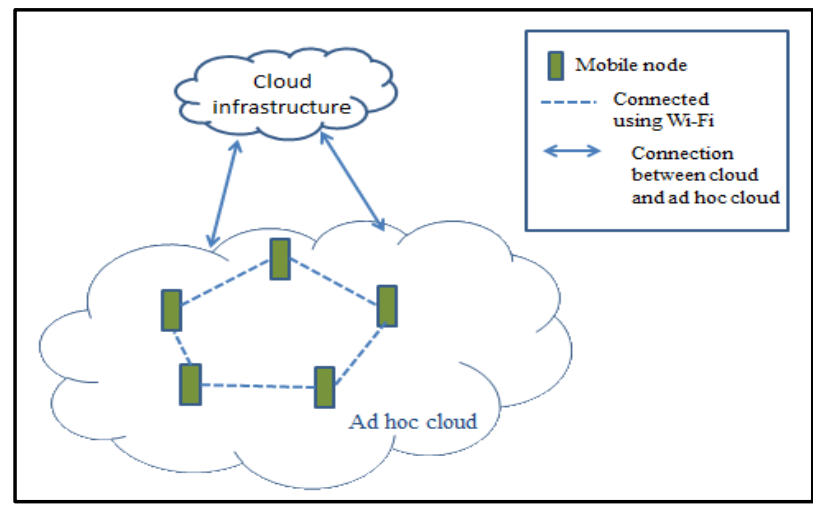

Figure1. Cloud Environment

Video downloading is one of the most popular applications on the mobile devices. Abundant energy is requisite for video download from the cloud by the mobile devices. The battery level is almost drained when a single mobile device attempts to download a file individually. Since a user expects the battery of the device to last for a longer period, energy efficiency of a mobile device is very vital. Hence there is a need for reducing the power consumption for video downloading applications. In practice the cloud user who wants to download a particular video gives request to the cloud. The cloud provider responds by offering the service and the user downloads it individually. In our proposed work, when a group of cloud users are interested in downloading the same file from the cloud simultaneously, they can engage in a group to collaboratively download the same file and share among the mobile users. In this case it is anticipated that the mobile devices should be in the proximity of each other. Providing uninterrupted service through the access of wireless medium is a major challenge [2]. Various frameworks have been developed for achieving seamless connectivity between the cloud and mobile device.

In this paperrelated work is reviewed in Section 2; Architecture and Proposed Work are presented in Section 3; Followed by the Conclusion in Section 4.

\section{RELATED WORKS}

Recently the growth of $3 \mathrm{~g}$ mobile networks increases rapidly after a slow start. They are being deployed on a broad scale all over the world which induces the mobile operators to provide various multimedia services. Video downloading is one such interesting multimedia application that utilizes the cloud infrastructure for the downloading process.Immense use of smart phones has made the mobile video to occupy the major portion of the total traffic. This significantly leads to high demand of processing power and battery constraints. Processing power of these mobile devices is considerably increasing to meet out the current trends. These factors promptly raise the mobile battery consumption and hence there is a need for the conservation of the energy level of the mobile battery.Various frameworks have been established to increase the mobile battery lifetime.

Ranjini Guruprasad suggested a methodology and an efficient algorithm where the optimal video rate is determined that eventually maximizes the life time of the mobile device during the video download process[3]. Battery load is considered as a major factor while designing these methodologies where minimizing the battery load and the duration of the load is the significant approach. Rate Mode Section Algorithm (RTMS) is used to monitor the battery load of the mobile devices. The power model is used to where the power requirements of the mobile device for video download are determined. The sum of the download power and the playback power gives the total energy consumption. Various scenarios have been taken into consideration for making an energy efficient video download. Taking these approaches, battery efficient video download process is carried out successfully 
Selim Ickin investigated the energy consumption of the mobile phones during the file downloading process [4]. He took the serialized and parallel scheduling algorithms as a scenario and conducted the experiment. A single smartphone is taken where a particular file is first downloaded via $3 \mathrm{~g}$ network and then the same file is downloaded via Wi-Fi tethering. Monsoon Power monitoring tool is used to measure the power consumption of the mobile devices and also the download duration taken for both the cases. The experiment is repeated by considering multiple factors and the sample results are analyzed. The results show that energy efficiency is greatly achieved when Wi-Fi tethering method is adopted.

The most appropriate transport technologies to deal with the simultaneous use of the same service by multiple users are multicasting and broadcasting [5]. This paper mainly focuses on the efficient file download process in the $3 \mathrm{~g}$ mobile networks. David Gómez-Barquero has made the file download process into three phases namely service advertisement, file transmission and post-delivery repair phase. Soft combining strategy is adopted where higher power gain is attained. Various factors like time, energy and number of users are considered and analyzed where efficient file download is obtained as a result.
Mohammad Reza Zakerinasab proposed a cloud assisted energy efficient system for smartphones involved in multimedia streaming applications[6]. He uses the cellular links and the Wi-Fi links simultaneously as the two communication channels. Light weight distributed scheduling algorithm is adopted for managing the collaboration and content sharing among the various nodes involved in the multimedia process. Two- level coding scheme is adopted for the downloading process in order to minimize the energy wastage. The experimental results show that up to $73 \%$ of the battery usage is saved by adopting this process for video streaming applications.

\section{PROPOSED WORK}

In our proposed work a group of mobile devices who are interested in downloading the same video at the same time form an ad hoc group. Each node downloads a segment of the video and finally exchange and reassembles the segments to get the whole file. Among all the nodes in the ad hoc group, segments are allocated only to the nodes with immense battery level while the others remain idle. But the complete file will be received by all the nodes of the ad hoc group once the download process is completed. Below is the Architecture diagram of the proposed work.

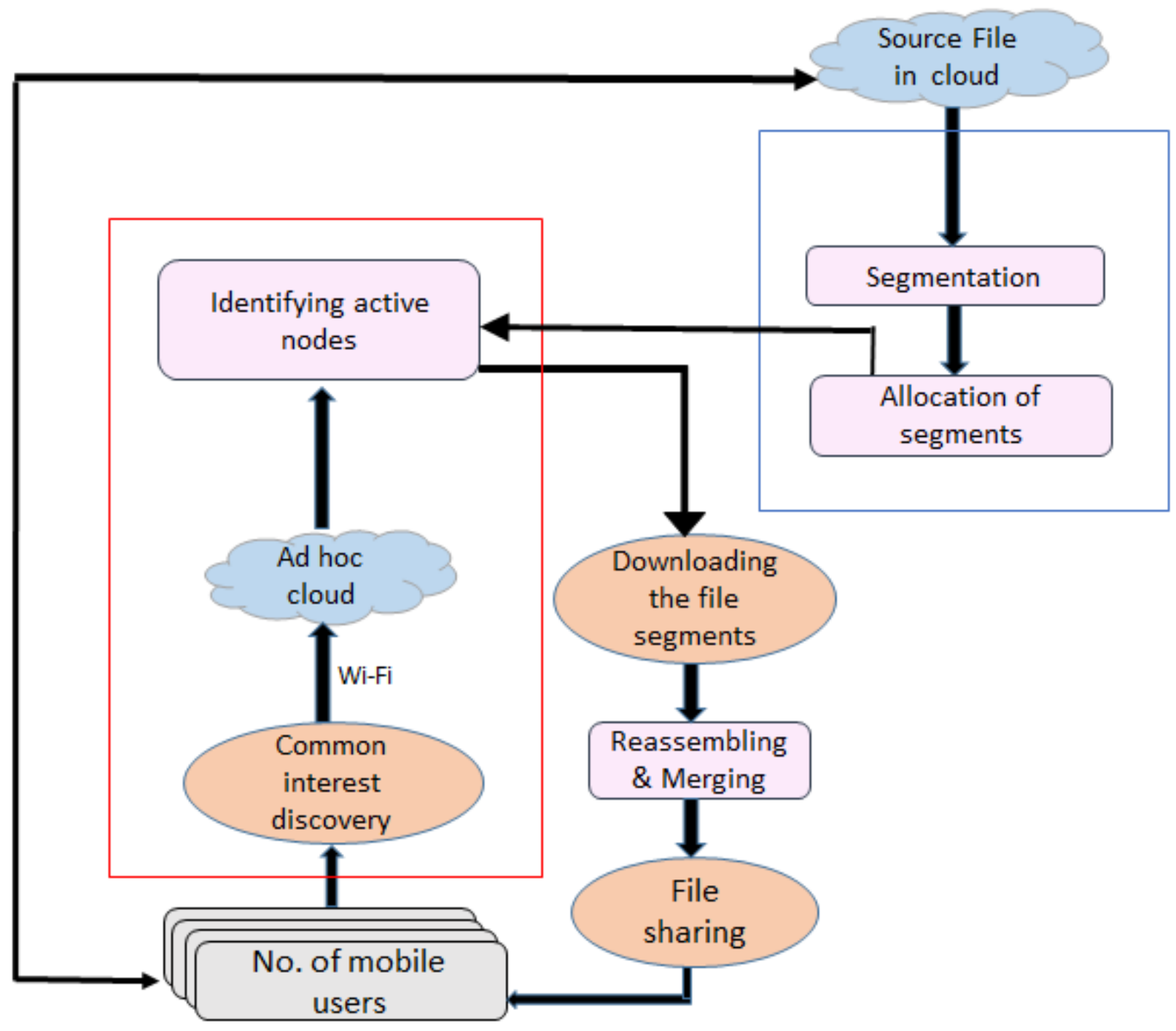

Figure2. Architecture of collaborative video download and sharing 


\subsection{Common Interest Discovery}

In Common Interest Discovery, the mobile users who are interested in downloading the same file at the same time are identified. The devices are commonly connected to the same Wi-Fi network and the one who initiates the process becomes the coordinator. The coordinator sends a request message to all the devices of the closed room to discover the common interest. The message can contain the file name or the URL of the file that the coordinator is interested to download. The others present in the same network can respond for the interest message. The devices that respond with the positive reply are made to join the ad hoc group. Thus all the mobile devices that are interested in downloading the same file at the same time are identified. These are the functionalities of the Common Interest Discovery Phase.

\subsection{Ad hoc Cloud Formation}

The mobile devices that are recognized with the common interest are intended to form the ad hoc group. Each of the nodes in the ad hoc group is connected to the common Wi-Fi connectivity. Since the temporary group (Ad hoc group) uses the services provided by the Cloud infrastructure, it is termed as 'Ad hoc Cloud'. The service offered by the cloud is effectively used by the members of the ad hoc cloud infrastructure.

\subsection{Energy Efficient Video Downloading \\ a) Battery Consideration}

Battery is one of the main constraints of the mobile devices. Video downloading is one of the most popular applications on the mobile devices. In order to download a video completely as an individual mobile, it might consume lot of energy. So, steps need to be taken care in order to reduce the battery consumption of the mobile devices in the downloading process. In our proposed work, as soon as the mobile device responds and states it common interest to download the same files from the Cloud the battery of the mobile devices monitored and the devices with considerable good battery level are identified. These identified nodes are named as 'Active Nodes'. These active nodes are identified so that these mobile devices can be involved in the downloading process. With this process compulsory participation of all the nodes in the group can be removed. So that the mobile devices with good battery level can only take part in the downloading process while the others with relatively low battery level are made idle in the group. This leads to the saving of power consumption of the mobile devices that remains idle in the Ad hoc Cloud. Once the file download is over, the file can be shared to all members of the group irrespective of the battery rate. This eliminates the compulsory participation of all devices in the group thus saving the energy of the mobile nodes. By this way video file can be downloaded by all the members of the group energy efficiently.

\section{b) Segmentation and Reassembly}

The identified active nodes involve the downloading process whereas the others remain idle in the group. The video in the cloud is segmented into as many segments as the number of active nodes present. Let us consider there are 10 active nodes that are identified in the Ad hoc cloud then the video source which is available in the cloud is partitioned into 10 segments. Let the segment be represented as $\mathbf{S}_{\mathbf{i}}$ If 10 actives nodes are identified in the Ad hoc Cloud then evidently the segments is represented as $\mathbf{S}_{\mathbf{1 0}}$, where $\mathrm{i}=\{1,2,3 \ldots 10\}$. Segments are allocated to each of the active nodes in the ad hoc cloud and they download their respective segment which is then sent to the coordinator who reassembles and merges those segments into a complete file.

In usual individual downloading method a mobile device has to download all the parts or a video file completely as an individual mobile. But in Collaborative download each node or mobile device are actually downloading only a part or a segment of the whole file instead of downloading the whole file. So it is advantageous that each mobile device downloads only a segment in order to download it as a whole but still they get the whole file downloaded by involving in the collaborative downloading process. This in turn also reduces the battery consumption of the mobile device because instead of downloading a complete file each node or mobile device actually downloads a segment of the file. Here Seamless connectivity can achieve by providing uninterrupted service through the access of wireless medium. If any node is unable to download a particular segment then steps need to be taken care such that the particular segment can be allocated to some other active node in the Ad hoc Cloud. So that process of downloading a video file from the cloud drives smoothly without any problems.

\subsection{File Sharing}

File sharing is the most important feature of this framework. Once the coordinator reassembles and merges all the segments of the video source to get the whole file, the sharing of the whole file to all the nodes of the ad hoc group takes place. The coordinator shares the complete file to all the members that includes the active nodes and also the idle nodes. Thus the active nodes by only downloading a part of the video source file gets the whole file being shared by coordinator. The idle nodes with relatively low battery level that do not involve in the downloading process also get the whole file being shared by coordinator. Thus the overall energy consumption for video download by all the nodes of the ad hoc group can be reduced to a greater extent by adopting collaborative video download method.

\section{CONCLUSION}

In this paper a framework is provided for group of users with common interest to collaboratively download a video file from the cloud and share it. In collaborative file download each node is expected to download only a segment of video file instead of downloading the complete file. These downloaded segments are then reassembled and merged into the whole file by the coordinator. This is then shared to all users of the ad hoc group. Only active nodes involve in segment download process whereas the idle nodes remain futile. This greatly reduces the battery consumption of the mobile devices as a whole. The collaborative downloading process provides a way to reduce the network traffic by avoiding repeated individual file download. Hence this framework is very helpful for the group of cloud users to download the same video file from the cloud and share it with less battery consumption, data consumption and time.

\section{REFERENCES}

[1] Olafur Ingthorsson."Data Center Knowledge", 2012, WEB:http://www.datacenterknowledge.com/archives/20 12/01/12/mobile-cloud-computing-will-soar-in-2012/

[2] Anuradha Ravi and Sateesh K. Peddoju, "Energy Efficient Seamless Service Provisioning in Mobile Cloud Computing" IEEE Seventh International Symposium on Service-Oriented System Engineering, 2013.

[3] Ranjini Guru Prasad and Sujit Dey, "Rate Adaption and Base station Reconfiguration for Battery Efficient Video 
Download", IEEE Wireless Communications and Networking Conference (WCNC): MAC, 2013.

[4] Selim Ickin, Thomas Zinner, Katarzyna Wac and Markus Fiedler, "Catching the Download Train: Energy efficient File Downloading On Smartphones", Proceedings of the $26^{\text {th }}$ International Teletraffic Congress (ITC), 2014.

[5] David Gomez-Barquero, Ana Fernandez-Aguilella and Narcis Cardona, "Multicast Delivery of File Download Services in Evolved 3G Mobile Networks With HSDPA and MBMS", IEEE Transactions On Broadcasting, Vol.55,No.4, December 2009.

[6] Mohammad Reza Zakerinasab and Mea Wang, "A Cloud-Assisted Energy-Efficient Video Streaming System for Smartphones", IEEE, 2013.

[7] Hoang T. Dinh, Chonho Lee, Dusit Niyato and Ping Wang, "A survey of mobile cloud computing: architecture, applications and approaches", Wireless Communication And Mobile Computing, October 2011. 